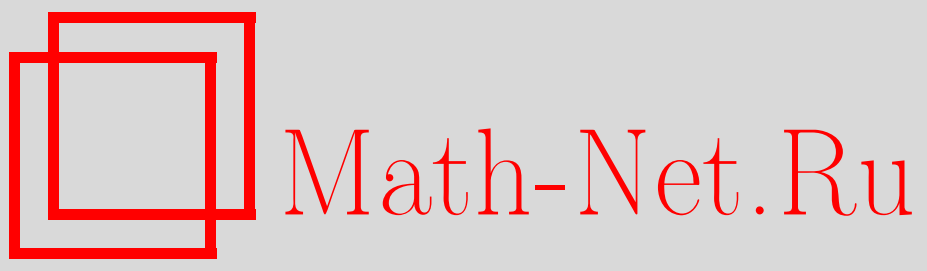

А. В. Косяк, Гауссовские меры на группе верхнетреугольных матриц бесконечного порядка, квазиинвариантные относительно обратного преобразования, Функи. анализ и его прил., 2000, том 34, выпуск $1,86-90$

DOI: https://doi.org/10.4213/faa287

Использование Общероссийского математического портала MathNet.Ru подразумевает, что вы прочитали и согласны с пользовательским соглашением

http://www.mathnet.ru/rus/agreement

Параметры загрузки:

IP : 54.209 .52 .79

26 апреля 2023 г., 16:52:26

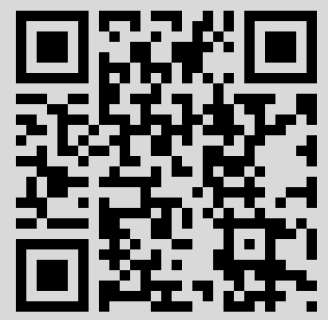


вершине находится точка минимума, если наружу — точка максимума, и если линия уровня проходит через вершину симплекса, рассекая угол, то в данной вершине расположена седловая точка. Случай прохождения линии уровня через вершину и одновременной перпендикулярности градиента одной из сторон симплекса является вырожденным (параметр принадлежит каустике).

Исходя из указанных правил, а также геометрических свойств касания прямой с окружностями и гиперболами (линиями уровня функций $U_{0}, U_{2}$ и $U_{1}$ соответственно) строятся характерные сечения каустики $[7,8]$. В случае каждого сечения $\mathbb{R}^{2}$ будет разбиваться на области, каждой из которых соответствует свой bif-расклад. Перебирая связные компоненты дополнений к этим сечениям и пользуясь описанными выше правилами, легко перечислить все возможные bif-расклады функций $U_{0}, U_{1}, U_{2}$ на всех допустимых симплексах, что дает в итоге полное описание возможных bif-раскладов для $W$ в виде указанных в теореме списков матриц.

ЗАмеЧАНИЕ. Назовем $\boldsymbol{l}=\langle\mathbf{M} \vec{\alpha}, \vec{\pi}\rangle+(-1)^{\nu}$, где $\vec{\alpha}=(1,-1,1), \vec{\pi}=(2,4,8)$, a $\mathbf{M}$ - матрица bif-расклада, обобщенным эйлеровым числом. Тогда для всех рассматриваемых случаев $\boldsymbol{l}=1$.

\title{
ЛитеРАТУРА
}

1. Струков Б. А., Леванюк А. П. Физические основы сегнетоэлектрических явлений в кристаллах. Наука, М., 1995. 2. Даринский Б. М., Сапронов Ю. И. Изв. ВУЗ, Матем., 41, № 2, 35-46 (1997). 3. Сапронов Ю. И. УМН, 51, вып. 1, 101-132 (1996). 4. Siersma D. Quart. J. Math. Oxford Ser. (2), 32, No. 125, 119-127 (1981). 5. Apнольд В. И., Варченко А. Н., Гусейн-Заде С. М. Особенности дифференцируемых отображений. Классификация критических точек, каустик и волновых фронтов. Наука, М., 1982. 6. Сапронов Ю. И. Полурегулярные угловые особенности гладких функций. Матем. сб., 180, № 10, 1299-1310 (1989). 7. Гнездилов А. В. Понтрягинские чтения-VIII, Тез. докл. Воронеж, ВГУ, 1997, с. 36. 8. Гнездилов А. В. Труды математического факультета ВГУ (новая серия), 2 (18), 19-26 (1997).

Воронежский государственный университет

Поступило в редакцию 30 марта 1998 г. В переработанном виде 26 марта 1999 г.

УДК 519.46

\section{Гауссовские меры на группе верхнетреугольных матриц бесконечного порядка, квазиинвариантные относительно обратного преобразования}

\author{
(c) 2000. А. В. Косяк
}

Для локально компактных групп $G$ известна [1] теорема о коммутанте:

Коммутант правого регулярного представления $\rho$ локально компактной группь $G$ порожден операторами левого регулярного представления $\lambda$, причем существует сплетаюиий оператор $J,(J f)(x)=\left(d h\left(x^{-1}\right) / d h(x)\right)^{1 / 2} \overline{f\left(x^{-1}\right)}$, такой, что $J \rho_{t} J=\lambda_{t}, t \in G$, где dh-левая мера Хаара. 
Впервые аналог регулярного представления бесконечномерных групп (групп токов) появился, по-видимому, в [2, 3]. В [4] доказана теорема о коммутанте для аналога регулярного представления группы токов, отвечающего мере Винера. Аналог регулярного представления для произвольной бесконечномерной топологической группы $G$, использующий $G$-квазиинвариантные меры $\mu$ на подходящих пополнениях $\widetilde{G}$ такой группы, определен в $[5,6]$.

Рассмотрим группу $B^{\infty}$ верхнетреугольных вещественных матриц бесконечного порядка, $B^{\infty}=\left\{x=I+x^{\prime}=I+\sum_{n=2}^{\infty} \sum_{k=1}^{n-1} x_{k n} E_{k n}\right\}$, и ее подгруппу «финитных» матриц $B_{0}^{\infty}=\left\{I+x^{\prime} \in B^{\infty} \mid x^{\prime}\right.$ финитна $\}$. Определим на группе $B^{\infty}$ гауссову меру $\mu_{b}$ следующим образом:

$$
d \mu_{b}(x)=\bigotimes_{k<n}\left(b_{k n} / \pi\right)^{1 / 2} \exp \left(-b_{k n} x_{k n}^{2}\right) d x_{k n}=\bigotimes_{k<n} d \mu_{b_{k n}}\left(x_{k n}\right)
$$

где $b=\left(b_{k n}\right)_{k<n}$ - двойная последовательность положительных чисел. Обозначим через $R_{t}(x)=x t$ правое действие, а через $L_{s}(x)=s^{-1} x$ левое действие группы $B^{\infty}$ на себе, и пусть $\Phi(x)=x^{-1}$ - преобразование перехода к обратной матрице. $\mathrm{B}[5,7]$ доказаны такие леммы:

ЛЕмма 1. $\mu_{b}^{R_{t}} \sim \mu_{b} \forall t \in B_{0}^{\infty}$.

ЛЕмма 2. $\mu_{b}^{L_{t}} \sim \mu_{b} \forall t \in B_{0}^{\infty} \Longleftrightarrow S_{k n}^{L}(b)=\sum_{m=n+1}^{\infty} b_{k m} b_{n m}^{-1}<\infty \forall k<n$.

Мы докажем следующую теорему.

Теорема 1. Если $E(b)=\frac{1}{8} \sum_{k<n} S_{k n}^{L}(b) b_{k n}^{-1}<\infty$, то $\mu_{b}^{\Phi} \sim \mu_{b}$. В этом случае определено правое регулярное представление $T^{R, \mu_{b}}$ и левое регулярное представление $T^{L, \mu_{b}}$ группь $B_{0}^{\infty}$ и справедлива теорема о коммутанте, причем оператор $J_{\mu_{b}}$ является сплетаюшим: $T_{t}^{L, \mu_{b}}=J_{\mu_{b}} T_{t}^{R, \mu_{b}} J_{\mu_{b}}, t \in B_{0}^{\infty}$, где $\left(J_{\mu_{b}} f\right)(x)=\left(d \mu_{b}\left(x^{-1}\right) / d \mu_{b}(x)\right)^{1 / 2} \overline{f\left(x^{-1}\right)}$.

ЗАмечАниЕ. Условие $E(b)<\infty$ является, видимо, также и необходимым условием эквивалентности $\mu_{b}^{\Phi} \sim \mu_{b}$.

СХемА доКАЗАТЕЛЬСТВА. Определим подгруппы $B_{n}, n=2,3, \ldots$, группы $B^{\infty}$ формулой $B_{n}=\left\{x \in B^{\infty} \mid x=I+\sum_{r<k, r \leqslant n} x_{r k} E_{r k}\right\}$. Пусть $\mu_{n}$ - проекция меры $\mu_{b}$ на подгруппу $B_{n}$. Имеем $d \mu_{n}(x)=\bigotimes_{r<k, r \leqslant n} d \mu_{b_{r k}}\left(x_{r k}\right)$. Заменим отображение $\Phi$ отображением $\widetilde{\Phi}=\Phi$ 。 $\theta$, где $\theta\left(I+x^{\prime}\right)=I-x^{\prime}, I+x^{\prime} \in B^{\infty}$. Так как меры $\mu_{b_{k n}}$ центрированы, то $\mu_{b}^{\widetilde{\Phi}} \sim \mu_{b} \Longleftrightarrow \mu_{b}^{\Phi} \sim \mu_{b}$. Определим однопараметрические группы преобразований $\widetilde{\Phi}^{t}, \widetilde{\Phi}_{n}^{t}, \tilde{\phi}_{n}^{t}, t \in \mathbb{R}$, группы $B^{\infty}$ следующим образом:

$$
\begin{gathered}
\widetilde{\Phi}^{t}\left(I+x^{\prime}\right)=I+\left(I-t x^{\prime}\right)^{-1} x^{\prime}, \quad \widetilde{\Phi}_{n}^{t}\left(I+x^{\prime}\right)=I+\left(I-t x^{\prime} e_{n}\right)^{-1} x^{\prime}, \\
\tilde{\phi}_{n}^{t}\left(I+x^{\prime}\right)=I+\left(I-t x^{\prime} e(n)\right)^{-1} x^{\prime},
\end{gathered}
$$

где $e_{n}=\operatorname{diag}(1, \ldots, 1,0, \ldots), e(n)=\operatorname{diag}(0, \ldots, 0,1,0, \ldots)$. Тогда $\widetilde{\Phi}=\widetilde{\Phi}^{1}, \widetilde{\Phi}^{t}=$ $\cdots \circ \tilde{\phi}_{n+1}^{t} \circ \tilde{\phi}_{n}^{t} \circ \tilde{\phi}_{n-1}^{t} \circ \cdots \circ \tilde{\phi}_{2}^{t}$ и $\widetilde{\Phi}_{n}^{t}=\tilde{\phi}_{n}^{t} \circ \tilde{\phi}_{n-1}^{t} \circ \cdots \circ \tilde{\phi}_{2}^{t}$. По группам $\tilde{\phi}_{n}^{t}$ построим однопараметрические группы унитарных операторов $U_{n}(t):\left(U_{n}(t) f\right)(x)$ 
$=\left(d \mu_{b}\left(\tilde{\phi}_{n}^{t}(x)\right) / d \mu_{b}(x)\right)^{1 / 2} f\left(\tilde{\phi}_{n}^{t}(x)\right)$, с генераторами $A_{n}$, определенные в пространстве $L_{2}\left(B^{\infty}, d \mu_{b}\right)$ при условии, что $\mu_{n}^{\tilde{\phi}_{n}^{t}} \sim \mu_{n}$ (см. лемму 3$)$. Имеем

$$
i A_{n}=\sum_{r=1}^{n-1} x_{r n} \sum_{m=n+1}^{\infty} x_{n m} D_{r m}=\sum_{r=1}^{n-1} x_{r n}\left(-i A_{r n}^{L}-D_{r n}\right),
$$

где $D_{r n}=\partial / \partial x_{r n}-b_{r n} x_{r n}$, а $A_{r n}^{L}$ - генераторы однопараметрических групп, отвечающих левому регулярному представлению, $i A_{r n}^{L}=d T_{I+t E_{r n}}^{L, \mu_{b}} /\left.d t\right|_{t=0}$. Оказывается, что группы $U_{n}(t)$ коммутируют, $\left[U_{n}(t), U_{m}(s)\right]=0$; поэтому $V_{n}(t)=$ $U_{n}(t) U_{n-1}(t) \cdots U_{2}(t)$ тоже является однопараметрической группой с генератором $\mathscr{A}_{n}=A_{n}+A_{n-1}+\cdots+A_{2}$ и действием

$$
\left(V_{n}(t) f\right)(x)=\left(\frac{d \mu_{b}\left(\widetilde{\Phi}_{n}^{t}(x)\right)}{d \mu_{b}(x)}\right)^{1 / 2} f\left(\widetilde{\Phi}_{n}^{t}(x)\right) .
$$

Пусть $U(t)$ - произвольная группа унитарных операторов, $A=A^{*}$ - ее генератор, т. е. $U(t)=\exp (i t A), f \in D(A)$ и $\|f\|=1$. Положим $F_{A}(t)=(U(t) f, f)$. Тогда

$$
\left|F_{A}(t)-1\right|^{2} \leqslant t^{2}\|A f\|^{2}, \quad t \in \mathbb{R} .
$$

Обозначим через $H(\mu, \nu)$ интеграл Хеллингера для мер $\mu$ и $\nu$ (см. [8, с. 99]). ЛЕмма 3. $\mu_{n}^{\tilde{\phi}_{n}} \sim \mu_{n} \Longleftrightarrow H\left(\mu_{n}^{\tilde{\phi}_{n}^{t}}, \mu_{n}\right)>0, t \in \mathbb{R} \backslash\{0\} \Longleftrightarrow\left\|A_{n} \mathbf{1}\right\|^{2}=$ $\frac{1}{8} \sum_{r=1}^{n-1} S_{r n}^{L}(b) b_{r n}^{-1}<\infty$.

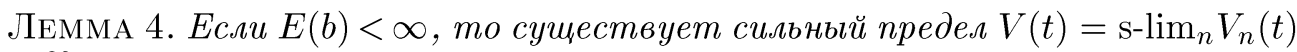
$=\prod_{n=2}^{\infty} U_{n}(t)$, являющийся однопараметрической группой унитарных операторов. В этом случае $\mu_{b}^{\tilde{\Phi}} \sim \mu_{b}$.

ДОКАЗАТЕЛЬСТВо ЛЕММЫ 3. В силу теоремы $1 \S 18$ из [9] и представлений

$$
\begin{aligned}
\mu_{n}^{\tilde{\phi}_{n}^{t}}(\Delta) & =\int_{\mathbb{R}^{n-1}} \bigotimes_{m=n+1}^{\infty} \mu_{(n, m)}^{\tilde{\phi}_{n, x}^{t}} \otimes \mu_{(n-1)}\left(\Delta_{x}\right) d \mu_{(n-1, n)}(x), \\
\mu_{n}(\Delta) & =\int_{\mathbb{R}^{n-1}} \bigotimes_{m=n+1}^{\infty} \mu_{(n, m)} \otimes \mu_{(n-1)}\left(\Delta_{x}\right) d \mu_{(n-1, n)}(x),
\end{aligned}
$$

где

$$
\begin{aligned}
& \mu_{(n, m)}=\bigotimes_{r=1}^{n} \mu_{b_{r m}}, \quad \mu_{(n-1)}=\bigotimes_{r<k<n} \mu_{b_{r k}}, \\
& \mu_{(n, m)}^{\tilde{\phi}_{n, x}^{t}}\left(x_{1 m}, x_{2 m}, \ldots, x_{n m}\right)=\bigotimes_{r=1}^{n-1} \mu_{b_{r m}}\left(x_{r m}+t x_{r n} x_{n m}\right) \otimes \mu_{b_{n m}}\left(x_{n m}\right), \\
& x=\left(x_{1 n}, x_{2 n}, \ldots, x_{n-1, n}\right), \quad \Delta=\bigcup_{x \in \mathbb{R}^{n-1}} \Delta_{x},
\end{aligned}
$$


имеем

$$
\begin{aligned}
\mu_{n}^{\tilde{\phi}_{n}^{t}} \sim \mu_{n} & \Longleftrightarrow \bigotimes_{m=n+1}^{\infty} \mu_{(n, m)}^{\tilde{\phi}_{n, x}^{t}} \sim \bigotimes_{m=n+1}^{\infty} \mu_{(n, m)} \quad \text { для п.в. } x\left(\bmod \mu_{(n-1, n)}\right) \\
& \Longleftrightarrow H\left(\bigotimes_{m=n+1}^{\infty} \mu_{(n m)}^{\tilde{\phi}_{n, x}^{t}}, \bigotimes_{m=n+1}^{\infty} \mu_{(n m)}\right)>0 \quad \text { для п.в. } x\left(\bmod \mu_{(n-1, n)}\right) .
\end{aligned}
$$

Окончательно, для п.в. $x\left(\bmod \mu_{(n-1, n)}\right)$ имеем при $\sum_{r=1}^{n-1} S_{r n}^{L}(b) b_{r n}^{-1}<\infty$

$$
H\left(\bigotimes_{m=n+1}^{\infty} \mu_{(n m)}^{\tilde{\phi}_{n, x}^{t}}, \bigotimes_{m=n+1}^{\infty} \mu_{(n m)}\right)=\prod_{m=n+1}^{\infty}\left(1+\frac{t^{2}}{4} \sum_{r=1}^{n-1} \frac{b_{r m}}{b_{n m}} x_{r n}^{2}\right)^{-1 / 2}>0
$$

Для доказательства леммы 4 покажем, что на плотном подмножестве $D=$ $\left\{\right.$ пространство многочленов от переменных $\left.\left(x_{k n}\right)_{k<n}\right\}$ в пространстве $H=$ $L^{2}\left(B^{\infty}, d \mu_{b}\right)$ имеется сходимость (где $\left.f \in D\right) \lim _{n+m, n \rightarrow \infty}\left(V_{n+m}(t)-V_{n}(t)\right) f=0$. В силу финитности функции $f \in D$ при некотором $p$ имеем $f=\mathbf{1} \otimes f_{(p)} \in$ $H^{(p)} \otimes H_{(p)}=H$, где

$$
\begin{gathered}
H_{(p)}=L^{2}\left(B_{(p)}, \mu_{(p)}\right), \quad H^{(p)}=L^{2}\left(B^{(p)}, \mu^{(p)}\right), \\
B_{(p)}=\left\{x \in B^{\infty} \mid x=I+\sum_{r<k \leqslant p} x_{r k} E_{r k}\right\}, \\
B^{(p)}=\left\{x \in B^{\infty} \mid x=I+\sum_{r<k, p<k} x_{r k} E_{r k}\right\}, \\
\mu_{(p)}=\bigotimes_{r<k \leqslant p} \mu_{b_{r k}}, \quad \mu^{(p)}=\bigotimes_{r<k, p<k} \mu_{b_{r k}} .
\end{gathered}
$$

Так как при $n \geqslant p$ имеем $\left(V_{n}(t)\right)^{-1} V_{n+m}(t)=\left[V_{n}(t)^{-1} V_{n+m}(t)\right]^{(p)} \otimes I_{(p)}$, где $[V]^{(p)}$ - проекция оператора $V \in L(H)$ на подпространство $H^{(p)} \otimes \mathbf{1}$ пространства $H=H^{(p)} \otimes H_{(p)}$, то в силу $(2)$

$$
\begin{aligned}
\left\|\left(V_{n+m}(t)-V_{n}(t)\right) f\right\|^{2} & =\left\|\left(\left[V_{n}(t)^{-1} V_{n+m}(t)\right]^{(p)} \otimes I_{(p)}-I^{(p)} \otimes I_{(p)}\right) \mathbf{1} \otimes f_{(p)}\right\|^{2} \\
& =\left\|\left(\left[V_{n}(t)^{-1} V_{n+m}(t)\right]^{(p)}-I^{(p)}\right) \mathbf{1}\right\|_{H^{(p)}}^{2}\left\|f_{(p)}\right\|_{H_{(p)}}^{2} \\
& \leqslant\left\|f_{(p)}\right\|_{H_{(p)}}^{2} t^{2}\left\|\mathscr{A}_{n, n+m} \mathbf{1}\right\|^{2}=\left\|f_{(p)}\right\|_{H_{(p)}}^{2} t^{2} \sum_{k=n+1}^{n+m}\left\|A_{k} \mathbf{1}\right\|^{2} \\
& =\left\|f_{(p)}\right\|_{H_{(p)}}^{2} \frac{t^{2}}{8} \sum_{k=n+1}^{n+m} \sum_{r=1}^{k-1} \frac{S_{r k}^{L}(b)}{b_{r k}} \rightarrow 0, \quad n \rightarrow \infty,
\end{aligned}
$$

где $\mathscr{A}_{n, n+m}$ - генератор однопараметрической группы унитарных операторов $\left(V_{n}(t)\right)^{-1} V_{n+m}(t)$. Таким образом,

$$
V(t)=\mathrm{s}-\lim _{n} V_{n}(t), \quad(V(t) f)(x)=\left(\frac{d \mu_{b}\left(\widetilde{\Phi}^{t}(x)\right)}{d \mu_{b}(x)}\right)^{1 / 2} f\left(\widetilde{\Phi}^{t}(x)\right)
$$


— унитарный оператор; поэтому $\int\left(d \mu_{b}\left(\widetilde{\Phi}^{t}(x)\right) / d \mu_{b}(x)\right) d \mu_{b}(x)=\|V(t) \mathbf{1}\|^{2}=$ $\|\mathbf{1}\|^{2}=1$. Следовательно (см. теорему $4 \S 15$ и замечание к теореме $1 \S 16$ из [9]), $\mu_{b}^{\tilde{\Phi}^{t}} \sim \mu_{b}$ и $\mu_{b}^{\tilde{\Phi}} \sim \mu_{b}$.

Автор выражает благодарность Ю. М. Березанскому за большое внимание к работе и рецензенту за ряд полезных замечаний.

\title{
ЛИТЕРАТУРА
}

1. Dixmier J. Les $C^{*}$-algebras et leur representations, Gautirs-Villars, Paris, 1969. 2. Исмагилов Р. С. Функц. анализ и его прил., 15, вып. 2, 73-74 (1981). 3. Albeverio S., Hoegh-Krohn R., Testard D. J. Funct. Anal., 41, 378-396 (1981). 4. Albeverio S., Hoegh-Krohn R., Testard D., Vershik A. J. Funct. Anal., 51, 115-131 (1983). 5. Kosyak A. V. Selecta. Math. Soviet., 11, 241-291 (1992). 6. Kosyak A. V. J. Funct. Anal., 125, 493-547 (1994). 7. Косяк A. В. Функц. анализ и его прил., 24, вып. 3, 82-83 (1990). 8. Го X.-C. Гауссовы меры в банаховых пространствах. Мир, М., 1976. 9. Скороход A. B. Интегрирование в гильбертовом пространстве. Наука, М., 1975.

\section{Проблема Рохлина о кратном перемешивании в классе действий положительного локального ранга*}

\author{
(c) 2000. В. В. Рыжиков
}

1. В. А. Рохлин [1] ввел в рассмотрение свойство кратного перемешивания и поставил вопрос, влечет ли за собой свойство перемешивания кратности 1 свойства перемешивания кратности $k>1$. Напомним, что автоморфизм $T$ пространства Лебега $(X, \mathscr{B}, \mu), \mu(X)=1$, обладает свойством перемешивания кратности $k$, если для любых множеств $A_{0}, \ldots, A_{k} \in \mathscr{B}$ при $\left|z_{p}-z_{q}\right| \rightarrow \infty$, $0 \leqslant p<q \leqslant k$,

$$
\mu\left(T^{z_{0}} A_{0} \cap T^{z_{1}} A_{1} \cap \cdots \cap T^{z_{k}} A_{k}\right) \longrightarrow \mu\left(A_{0}\right) \mu\left(A_{1}\right) \cdots \mu\left(A_{k}\right) .
$$

Говорят, что автоморфизм $T$ обладает локальным рангом $\beta>0$, если для некоторой последовательности $U_{j}=\bigsqcup_{k \in Q_{j}} T^{z} B_{j}$ башен Рохлина-Халмоша, где $Q_{j}=\left\{0,1, \ldots, h_{j}\right\}$, выполнены следующие условия: $\mu\left(U_{j}\right) \rightarrow \beta$ и для каждого $A \in \mathscr{B}$ пересечение $U_{j} \cap A$ асимптотически близко к объединению некоторого набора этажей в башне. Это означает, что $\mu\left(\left(U_{j} \cap A\right) \Delta \bigsqcup_{z \in S_{j}} T^{z} B_{j}\right) \rightarrow 0$ при $j \rightarrow \infty$ для некоторой последовательности множеств $S_{j} \subset\{0,1, \ldots, h(j)-1\}$ (зависящих от $A)$. Для $\mathbb{Z}^{n}$-действий и $\mathbb{R}^{n}$-действий (потоков) определение аналогично, причем конфигурации $Q_{j}$ имеют вид

$Q_{j}=\left\{0,1, \ldots, h_{1}(j)\right\} \times \cdots \times\left\{0,1, \ldots, h_{n}(j)\right\}, \quad h_{m}(j) \rightarrow \infty, j \rightarrow \infty, 1 \leqslant m \leqslant n$. 